\title{
Macroscopic Signature of Protected Spins in a Dense Frustrated Magnet
}

\author{
S. Ghosh, ${ }^{1, *}$ T. F. Rosenbaum, ${ }^{2}$ and G. Aeppli ${ }^{3}$ \\ ${ }^{1}$ School of Natural Sciences, University of California, Merced, California 95343, USA \\ ${ }^{2}$ James Franck Institute and Department of Physics, University of Chicago, Chicago, Illinois 60637, USA \\ ${ }^{3}$ London Centre for Nanotechnology and Department of Physics and Astronomy, UCL, London, WC1E 6BT, United Kingdom
} (Received 23 April 2008; revised manuscript received 18 August 2008; published 10 October 2008)

The inability of systems of interacting objects to satisfy all constraints simultaneously leads to frustration. A particularly important consequence of frustration is the ability to access certain protected parts of a system without disturbing the others. For magnets such "protectorates" have been inferred from theory and from neutron scattering, but their practical consequences have been unclear. We show that a magnetic analogue of optical hole-burning can address these protected spin clusters in a well-known, geometrically frustrated Heisenberg system, gadolinium gallium garnet. Our measurements additionally provide a resolution of a famous discrepancy between the bulk magnetometry and neutron diffraction results for this magnetic compound.

PACS numbers: 75.40.Gb, 75.45.+j, 75.50.Dd, 75.50.Lk

One important property of frustrated systems is that the number of most probable (lowest energy) configurations is not finite and grows exponentially with the system size, resulting in finite zero-temperature ( $T$ ) entropy. A number of real magnets, which are among the simplest physical realizations of frustrated systems exhibit nonzero entropy [1-3] as $T \rightarrow 0$. What has been missing is a simply measured, macroscopic, dynamical effect associated directly with decoupled spin subsets. We report here the discovery of such an effect in a dense, ordered network of spins. Our result shows that spins can be "protected" [4] from each other in a dense magnetic medium. It also provides a sharp distinction between a well-known magnet, gadolinium gallium garnet, where the incompatible constraints are due to lattice geometry, and conventional spin glasses, where they are due to disorder.

The archetypical frustrated magnet is the triangular antiferromagnet (AFM) [Fig. 1(a)] consisting of Ising spins. If, on a particular triangle, a spin is antiparallel to its two neighbors, up and down orientations have equal energy. This spin is a protected degree of freedom because it can flip as if it were decoupled from its environment. Such degrees of freedom involving larger groupings of spins can be found for more complicated lattices [Fig. 1 (b)] [5-8]. Celebrated 3D generalizations of the Kagome lattice include pyrochlores [9] and garnets. Here we focus on Gadolinium Gallium Garnet $\left(\mathrm{Gd}_{3} \mathrm{Ga}_{5} \mathrm{O}_{12}\right.$ or GGG), a cubic compound containing magnetic $\mathrm{Gd}$ ions on cornersharing triangles that form two interpenetrating networks [Fig. 1(c)]. The Weiss temperature $\Theta_{\text {weiss }}$, proportional to a combination $J$ of the exchange $(1.5 \mathrm{~K})$ and dipolar $(\sim 0.7 \mathrm{~K})$ couplings, is approximately $2 \mathrm{~K}[10]$. The single ion anisotropy is less than $0.040 \mathrm{~K}$, making this a Heisenberg system. The geometric frustration postpones magnetic order to $T<0.180 \mathrm{~K}$, where the bulk magnetic response [11] begins to resemble that of a magnetic glass. However, muon spin relaxation does not display the be- havior conventionally associated with spin glasses below $T_{g}$ [12]. Neutron scattering [13] reveals sharp magnetic diffraction peaks superposed on a spin liquidlike structure factor. Together with recent theory [14], these suggest that in the pure limit, conventional long range AFM order, rather than a disordered spin glass, coexists with the spin fluid that arises from local degrees of freedom as illustrated in Fig. 1(c).

To probe the true nature of the ground state [Fig. 2(a)] we measured the dynamic magnetization $M$ for oscillating

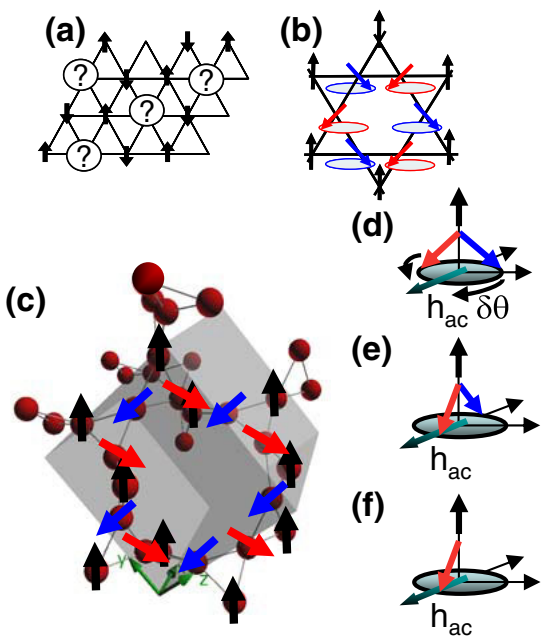

FIG. 1 (color online). (a) Spins at vertices of a triangular Ising antiferromagnetic (AFM) lattice (labeled "?") are geometrically frustrated (b) Heisenberg AFM on a Kagome lattice. The "local" degree of freedom corresponds to a collective rotation of the red and blue spins around the black spin. (c) One of the Gd sublattices of GGG, showing the ten-membered ring of spins which can rotate about the black spin direction, forming a local degree of freedom analogous to that in (b). (d)-(f) Schematics of spin triads to understand response of local degrees of freedom to small external fields $h_{\text {ac }}$. The black spin sublattice is assumed to be rigid. (a) and (b) are adapted from [7]. 
(a)

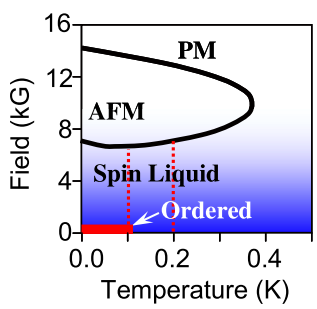

(b)

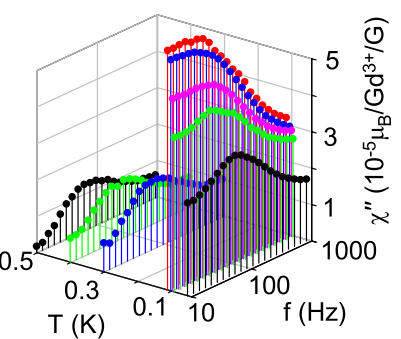

(c)

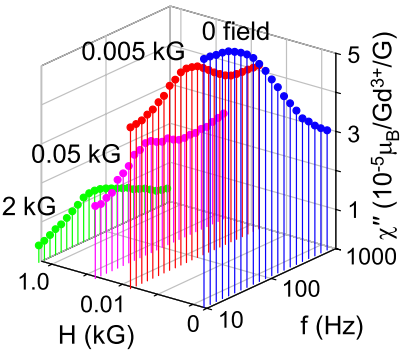

FIG. 2 (color online). (a) Phase diagram (following [11]). Our study focuses on the spin liquid phase with $H<4 \mathrm{kG}<6 \mathrm{kG}$. The red dashed lines indicate the temperatures along which we have conducted detailed dc $(H)$ studies. (b) $\chi^{\prime \prime}$, as a function of $T$ and $f$ at $H=0$ in the linear regime. (c) $H$ dependence of $\chi^{\prime \prime}$. The low $f$ plateau in $\chi^{\prime \prime}(f)$ connected to spin ordering at $0.100 \mathrm{~K}$ disappears rapidly with the application of $H$.

drive fields $h_{\mathrm{ac}} \cos (2 \pi f t)$ down to $T=0.050 \mathrm{~K}$ as a function of the amplitude $h_{\mathrm{ac}}$ and frequency $f$. The sample is a single crystal of GGG (long axis [100]) grown by the Czochralski method and obtained from Princeton Scientific. In the limit of small $h_{\mathrm{ac}}, M$ is linear in $h_{\mathrm{ac}}$ and the susceptibility $\chi(f)=M / h_{\mathrm{ac}}$. In Fig. 2(b) we plot the imaginary part $\chi^{\prime \prime}(f)$ against $f$ and $T$ for $h_{\mathrm{ac}}=0.04 \mathrm{G}$, which we have established to be in the linear regime. At the high-temperature end $\left(0.500 \mathrm{~K}\right.$, black), $\chi^{\prime \prime}(f)$ has a peak at $80 \mathrm{~Hz}$ with a FWHM of $\sim 1.3$ decades in $f$, implying that the dynamic response is described by a distribution of relaxation times [15]. As $T$ is lowered, the peak height of $\chi^{\prime \prime}(f)$ increases while the basic shape and $f_{\text {peak }}$ remain almost the same, until $0.110 \mathrm{~K}$, where the shape alters drastically. There is no longer a peak in $\chi^{\prime \prime}(f)$, which plateaus at low $f$. The flat, $f$-independent tail for $f \rightarrow 0$ originates from $1 / f$ noise in $M$, which is characteristic of the dynamical scale invariance characteristic of second order phase transitions as well as certain ordered phases with complex hierarchies of ground states, such as spin glasses [16]. Cooling below $0.100 \mathrm{~K}$ reduces $\chi^{\prime \prime}(f)$ and its shape returns to that seen above $0.110 \mathrm{~K}$, again with $f_{\text {peak }}=80 \mathrm{~Hz}$. This is neither expected nor seen for con- ventional spin glasses [16]. It looks more like a transition to a conventional ordered state, where there is critical slowing down and condensation of slow modes at the phase transition, below which the excitations harden as the order grows. The picture given by the linear susceptibility is consistent with neutron scattering data [13], where relatively sharp magnetic diffraction peaks correspond to partial AFM order for $T<T_{N}=0.100 \mathrm{~K}<0.140 \mathrm{~K}$. However, our bulk measurements reveal something unusual, namely, that the frequency below which a scaleinvariant response is observed corresponds to an energy $0.3 \mathrm{peV} \ll k_{B} T_{N} \sim 10 \mu \mathrm{eV} \ll J \sim 200 \mu \mathrm{eV}$. For an ideal soft mode transition, all of these energy scales would be of the same order. However, soft modes are often accompanied by a "central peak" [17], typically thought to be a contribution of very slowly relaxing clusters of ordered material in a disordered matrix above $T_{N}$.

Given the previous work [11] on the glasslike behavior of GGG, it is natural to ask whether our data are in the linear regime. Figures 3(a)-3(d) show measurements of $M\left(h_{\mathrm{ac}}\right)$ for $f=29.9 \mathrm{~Hz}$ at a variety of temperatures. At $0.4 \mathrm{~K}$ we are clearly in the linear regime while at $T_{N}=$ $0.100 \mathrm{~K}$, there are obvious signs of a crossover from a large, low- $h_{\mathrm{ac}}$ response to a substantially smaller high- $h_{\mathrm{ac}}$ magnetic response. At the same time, $M^{\prime \prime}$ has a strong peak, which means that dissipation is reduced on going into the high- $h_{\mathrm{ac}}$ regime. The highly nonlinear response of

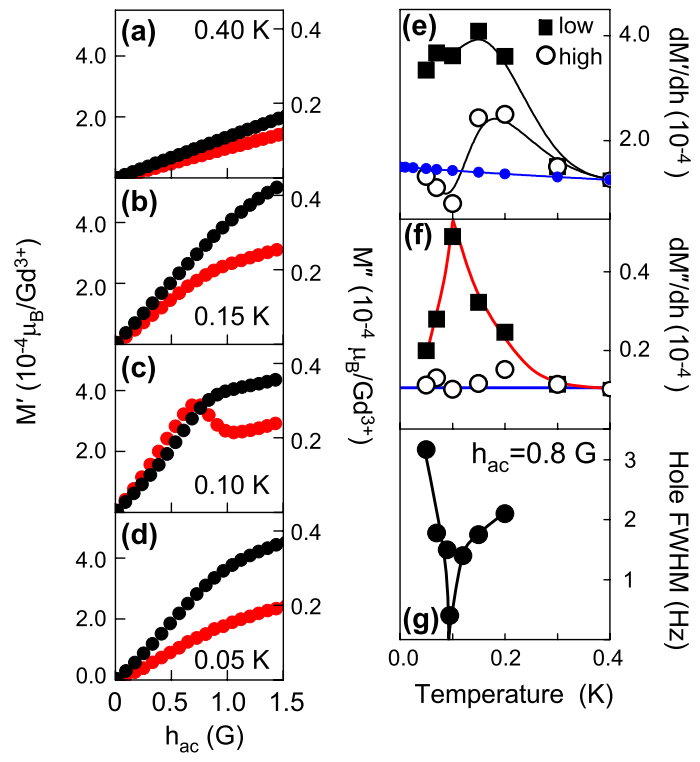

FIG. 3 (color online). (a)-(d) Real ( $M^{\prime}$, black, left axis) and imaginary $\left(M^{\prime \prime}\right.$, red, right axis) parts of the magnetization as a function of $h_{\mathrm{ac}}$ at different $T$ for $f=29.9 \mathrm{~Hz}$. (e) The $T$ dependence of $d M^{\prime} / d h_{\mathrm{ac}}$ at low $\left(h_{\mathrm{ac}}=0.04 \mathrm{G}\right)$ and high $\left(h_{\mathrm{ac}}=\right.$ $1.4 \mathrm{G})$ drive fields. The line (blue, with solid circles) corresponds to the Curie-Weiss law representing the extrapolated high-temperature susceptibility dominated by the frustrated units of GGG. (f) The analogous plot for $M^{\prime \prime}$. (g) The spectral width of the hole in the susceptibility as a function of temperature. All lines are guides to the eye. 
GGG revealed at low $h_{\mathrm{ac}}$ in the current experiment clearly needs to be taken into account when analyzing magnetic susceptibility data. Indeed, the AFM phase transition seen in the neutron experiments and in our linear susceptibility data can be masked by the nonlinearities which clearly matter for ac magnetometry with $h_{\mathrm{ac}}=1 \mathrm{G}$ [11].

Similar behavior [18] has been seen in the dilute Ising salt $\mathrm{LiHo}_{x} \mathrm{Y}_{1-x} \mathrm{~F}_{4}$, but with the crucial difference that there, at high $h_{\mathrm{ac}}, M^{\prime \prime} \rightarrow 0$ and saturation and phase locking are complete. By contrast, in concentrated GGG there is a persistent linear (in $h_{\mathrm{ac}}$ ) background. Because we were unable to derive an analytic expression for $M\left(h_{\mathrm{ac}}\right)$, we have simply evaluated the low and high- $h_{\mathrm{ac}}$ derivatives, $d M^{\prime} / d h_{\mathrm{ac}}$ and $d M^{\prime \prime} / d h_{\mathrm{ac}}$, as a function of $T$, plotted in Figs. 3(e) and 3(f). The high $h_{\mathrm{ac}}$ response shows a strong peak above the ordering temperature, moving towards the extrapolated (from high $T$ ) Curie-Weiss susceptibility [10] of GGG, while the low $h_{\text {ac }}$ response has its sharp maximum at $T_{N}$, with an amplitude corresponding to $10 \%$ of the $\mathrm{Gd}$ ions rotating freely.

When faced with a new dynamical phenomenon it is important to determine whether it is due to the relaxation of many coupled spins, or to the motion of smaller groups of decoupled spins. The classic experimental method for discrimination between the two possibilities is hole burning [19]. We apply simultaneously a sinusoidal pump, with amplitude $0.8 \mathrm{G}$, sufficient to approach the crossover to the high $h_{\text {ac }}$ regime in Figs. 3(a)-3(d), and a small amplitude probe $(0.04 \mathrm{G})$. Figure $4(\mathrm{a})$ shows how at $0.150 \mathrm{~K}$, the pump carves out a hole in $\chi^{\prime \prime}(f)$, centered at $f_{\text {pump }}$ and with FWHM $\sim 3 \mathrm{~Hz}$. No other part of $\chi^{\prime \prime}(f)$ is similarly affected although at $f<250 \mathrm{~Hz}$ the entire spectrum is attenuated relative to that observed without pumping. The holes contain about $0.5 \%$ of the total spectral weight seen at $0.150 \mathrm{~K}$, a macroscopic number $\sim 10^{17}$ spins $\mathrm{cm}^{-3}$. We can burn similar holes at any frequency $f<f_{\text {peak }}$. At $0.095 \mathrm{~K}$ [Fig. 4(b)], just below $T_{N}$, we see a sharper, deeper hole, which then becomes broader and shallower well within the ordered phase, at $0.050 \mathrm{~K}$. Figure $3(\mathrm{~g})$ shows how the spectral hole width, inversely proportional to the quality $Q$ of the oscillators responsible for the excitations at $29.9 \mathrm{~Hz}$, has a sharp minimum at $T_{N}$. We have also applied a dc bias field $H$ to approach the different AFM phase [see Fig. 2(c)] revealed previously above $H=6 \mathrm{kG}$. As shown in Fig. 4(e), the sharp hole is robust up to at least $0.5 \mathrm{kG}$, but it is greatly broadened and suppressed at $2 \mathrm{kG}$ and unrecognizable at $4 \mathrm{kG}$.

What could give rise to the apparent high- $Q$ oscillators in a dense frustrated magnet such as GGG? Most likely are the red and blue spin clusters, shown in Fig. 1(c), which can be rotated at no cost relative to an ordered, surrounding backbone of black spins. A small external field, such as $h_{\mathrm{ac}}$, will break this rotational symmetry as long as it is not parallel to the field produced by the backbone. In particular, once the anisotropy field $h_{A}$ is exceeded, there will be a spin flop, shown schematically for the spin triad in Fig. 1
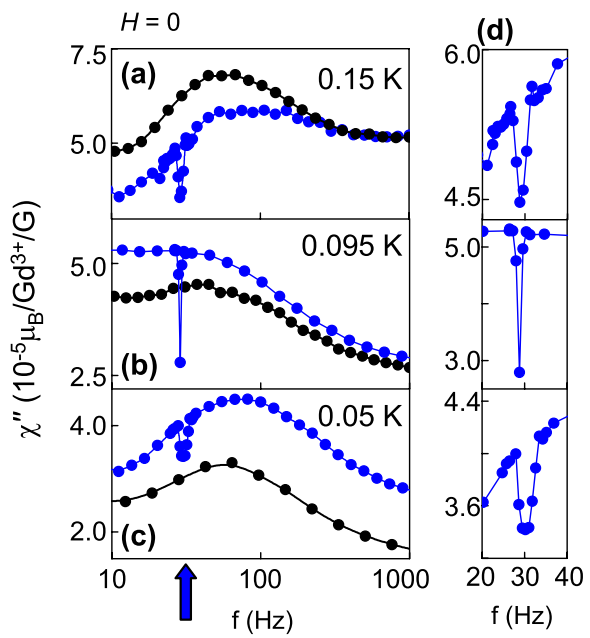

$T=0.100 \mathrm{~K}$

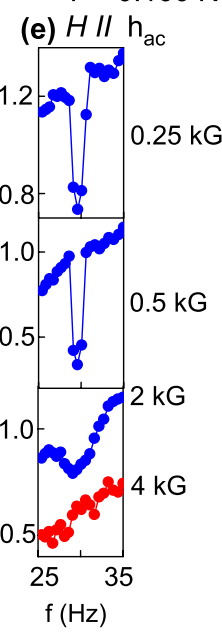

FIG. 4 (color online). (a)-(c) $\chi^{\prime \prime}$ as a function of $f$ at different $T$ bracketing the ordering temperature in the linear (black) and nonlinear (blue) regimes. The nonlinear susceptibility is measured with a pump-probe combination. $f_{\text {pump }}=29.9 \mathrm{~Hz}$ (blue arrow). (d) The holes in (a)-(c) magnified, sharpest at ordering. (e) Hole-burning at $0.100 \mathrm{~K}$ at nonzero $H$. The ability to burn holes disappears by $H=4 \mathrm{kG}$ [data shown in the bottom panel of (e) with an added offset for clarity].

(d), with the red and blue spins at an angle of $30^{\circ}$ relative to the black spin and simultaneously orthogonal to $h_{\mathrm{ac}}$. There will then be some small additional rotation $(\delta \theta)$ of the red and blue spins towards each other and $h_{\mathrm{ac}}$. In the pure limit, where there are no random internal fields, there will be a much smaller response [Fig. 1(e)] for $h_{\mathrm{ac}}<h_{A}$ than for $h_{\mathrm{ac}}>h_{A}$. Indeed, the imaginary part of the bulk response should be rigorously zero [20] for $h_{\mathrm{ac}} \rightarrow 0$ for a clean Heisenberg magnet (with $h_{A}=0$ ) consisting of AFM triangles.

Disorder changes the situation markedly and the response can be stronger for $h_{\mathrm{ac}}<h_{A}$ than for $h_{\mathrm{ac}}>h_{A}$. For example, if there is a missing magnetic atom on the ten-membered ring of Fig. 1(c), the ring will have a net, uncanceled moment $M_{u}$ which is orthogonal to the black spins, and before the spin-flop transition occurs the main effect of small $h_{\mathrm{ac}}$ will be to orient $M_{u}$, a situation which can be understood by simply removing one spin from Fig. 1(e) to obtain Fig. 1(f). Above $h_{A}$, the spin-flop transition will still occur and $M_{u}$ will be perpendicular to $h_{\text {ac }}$, but with a correspondingly reduced magnetic response $d M / d h_{\mathrm{ac}}$. This leads to precisely what we observe in Fig. 3. Dissipation is reduced at the crossover between the spin-flop regimes because continuous rotation of moments orthogonal to $h_{\mathrm{ac}}$, as illustrated in Fig. 1(d), is intrinsically barrier-free, in contrast to the flipping of moments for $h_{\mathrm{ac}}<h_{A}$ as the applied field oscillates.

We can estimate the anisotropy field $h_{A}$ to be close to the value of $h_{\mathrm{ac}}$ where the susceptibility crosses between the linear and nonlinear regimes. From Fig. 3 this is approximately $0.8 \mathrm{G} . h_{A}$ also leads to an understanding of $f_{\text {peak }}$ 
which corresponds to the most probable relaxation rate, typically of order $J$, translating to $50 \mathrm{GHz}$ for GGG. Because we are dealing with the relaxation of groups of spins rather than individual moments, the observed $f_{\text {peak }}=$ $80 \mathrm{~Hz}$ is much smaller. In this case, the relaxation rate can be estimated using a WKB approximation [21] as $f_{\text {peak }}=$ $f_{0} e^{-S / \hbar}$, with the tunneling action $S / \hbar=N s \sqrt{2 k / \Delta E}$ for magnetic clusters with $N$ spins of magnitude $s$, an anisotropy energy $k$, and an energy barrier $\Delta E$, due, e.g., to interactions with neighboring clusters [21]. If we set the attempt frequency $f_{0}=J$ and $N=10$, the ring size of Fig. 1(c), we can obtain $f_{\text {peak }}=80 \mathrm{~Hz}$, in agreement with the experiment provided that $2 k / \Delta E \sim 1 / 3$. If we take $k=$ $0.4 \mathrm{mK}=g s \mu_{B} h_{A}$, then $\Delta E \sim 3 \mathrm{mK}$, which is of the order of the next nearest neighbor magnetic interaction [10].

Inevitable longer-range interactions [14], beyond individual triangles, fill out the picture. In particular, at the lowest temperatures the fluctuating, uncompensated moments coexist with the unsaturated AFM order revealed by neutrons, and via the further neighbor interactions, display dynamics conditioned by the static mean field from the background antiferromagnetism. If we impose static ferromagnetic correlations via an external field, we see the same reduction in $Q$ that is produced by lowering $T$ below $T_{N}$ for $H=0$. At $T_{N}$, the static mean field from the other clusters is by definition zero, meaning that the defect-centered clusters are truly protected and independent of the AFM backbone, so that here we obtain the highest possible $Q$. On further warming, the fluctuation rates of the backbone move through the hole-burning frequency, coupling all modes together, and so $Q$ deteriorates again.

We have produced an experimental resolution of the famous discrepancy between bulk magnetometry [11] and magnetic neutron diffraction [13] for GGG. The transition temperature identified previously as the entry point to a spin glass is actually above the condensation temperature $T_{N}$ for a continuum of ultrasoft modes in the low frequency, linear magnetic response. Completely contrary to conventional phase transitions where modes are maximally coupled at a phase transition, at $T_{N}$, these oscillators achieve maximum decoupling as measured by the quality factors established by magnetic hole burning. Our discovery that the characteristic frequency $f_{\text {peak }}$ is independent of $T$ and so is not thermally activated, indicates that the protected, defect-nucleated degrees of freedom are actually behaving as quantum rather than classical objects.

The work at the University of Chicago was supported by DOE BES under Grant No. DE-FG02-99ER45789. G. A. acknowledges support from the Wolfson Foundation, EPSRC and the Rockefeller Foundation for a stay devoted to this manuscript at the Bellagio Study Center.

*sghosh@ucmerced.edu
[1] Steven T. Bramwell and Michel J. P. Gingras, Science 294, 1495 (2001).

[2] A. P. Ramirez, A. Hayashi, R. J. Cava, R. Siddharthan, and B. S. Shastry, Nature (London) 399, 333 (1999).

[3] A. P. Ramirez and R. N. Kleiman, J. Appl. Phys. 69, 5252 (1991).

[4] R. B. Laughlin and D. Pines, Proc. Natl. Acad. Sci. U.S.A. 97, 28 (2000); L. B. Ioffe, M. V. Feigel'man, A. Ioselevich, D. Ivanov, M. Troyer, and G. Blatter, Nature (London) 415, 503 (2002); Alexei Kitaev, arXiv:cond-mat/ 0609441v2; M.E. Zhitomirsky and Hirokazu Tsunetsugu, Phys. Rev. B 75, 224416 (2007); Andreas Mielke and Hal Tasaki, Commun. Math. Phys. 158, 341 (1993); A Mielke, J. Phys. A 24, 3311 (1991); M.E. Zhitomirsky and Hirokazu Tsunetsugu, Phys. Rev. B 70, 100403(R) (2004); J. Richter, J. Schulenburg, A. Honecker, J. Schnack, and H.-J. Schmidt, J. Phys. Condens. Matter 16, S779 (2004).

[5] K. Husimi and I. Syôzi, Prog. Theor. Phys. 5, 177 (1951).

[6] X. Obradors, A. Labarta, A. Isalgué, J. Tejada, J. Rodriguez, and M. Pernet, Solid State Commun. 65, 189 (1988).

[7] G. Aeppli and P. Chandra, Science 275, 177 (1997).

[8] S.-H. Lee, C. Broholm, W. Ratcliff, G. Gasparovic, Q. Huang, T.H. Kim, and S.-W. Cheong, Nature (London) 418, 856 (2002).

[9] Y. Taguchi, Y. Ohhara, H. Yoshizawa, N. Nagaosa, and Y. Tokura, Science 291, 2573 (2001).

[10] W.I. Kinney and W.P. Wolf, J. Appl. Phys. 50, 2115 (1979).

[11] P. Schiffer et al., Phys. Rev. Lett. 74, 2379 (1995).

[12] S. R. Dunsiger et al., Phys. Rev. Lett. 85, 3504 (2000); I. M. Marshall et al., J. Phys. Condens. Matter 14 , L157 (2002).

[13] O. A. Petrenko, C. Ritter, M. Yethiraj, and D. McK. Paul, Phys. Rev. Lett. 80, 4570 (1998).

[14] T. Yavors'kii, M. Enjalran, and M. J. P. Gingras, Phys. Rev. Lett. 97, 267203 (2006).

[15] P. Debye, Polar Molecules ('The Chemical Catalogue Company Inc., New York, 1929).

[16] M. H. Occio, H. Bouchiat, and P. Monod, J. Magn. Magn. Mater. 54, 11 (1986); W. Wu, B. Ellman, T. F. Rosenbaum, G. Aeppli, and D.H. Reich, Phys. Rev. Lett. 67, 2076 (1991).

[17] J.D. Axe, S. M. Shapiro, G. Shirane, and T. Riste, in Proceedings of the NATO Advanced Study Institute on Anharmonic Lattices, Structural Phase Transitions, and Melting, Geilo, Norway, 1973, edited by T. Riste (Noordhoff, Leiden, 1974); R.W. Youngblood, G. Aeppli, J.D. Axe, and J. A. Griffin, Phys. Rev. Lett. 49, 1724 (1982).

[18] S. Ghosh, R. Parthasarathy, T.F. Rosenbaum, and G. Aeppli, Science 296, 2195 (2002).

[19] Persistent Spectral Hole Burning: Science and Applications, edited by W.E. Moerner (Springer-Verlag, New York, 1988).

[20] S.-H. Lee et al., Europhys. Lett. 35, 127 (1996).

[21] O. G. Shpyrko et al., Nature (London) 447, 68 (2007); B. Barbara et al., J. Appl. Phys. 73, 6703 (1993); O. Waldmann, C. Dobe, H. Mutka, A. Furrer, and H.U. Güdell, Phys. Rev. Lett. 95, 057202 (2005). 